\title{
Investigation of deep indoor NB-IoT propagation attenuation
}

Malarski, Krzysztof Mateusz; Thrane, Jakob; Bech, Markus Greve; Macheta, Kamil; Christiansen, Henrik Lehrmann; Petersen, Martin Nordal; Ruepp, Sarah Renée

\section{Published in:}

Proceedings of 2019 IEEE 90th Vehicular Technology Conference

Link to article, DOI:

10.1109/vtcfall.2019.8891414

Publication date:

2019

Document Version

Peer reviewed version

Link back to DTU Orbit

Citation (APA):

Malarski, K. M., Thrane, J., Bech, M. G., Macheta, K., Christiansen, H. L., Petersen, M. N., \& Ruepp, S. R. (2019). Investigation of deep indoor NB-loT propagation attenuation. In Proceedings of 2019 IEEE 90th Vehicular Technology Conference [8891414] IEEE. https://doi.org/10.1109/vtcfall.2019.8891414

\section{General rights}

Copyright and moral rights for the publications made accessible in the public portal are retained by the authors and/or other copyright owners and it is a condition of accessing publications that users recognise and abide by the legal requirements associated with these rights.

- Users may download and print one copy of any publication from the public portal for the purpose of private study or research.

- You may not further distribute the material or use it for any profit-making activity or commercial gain

- You may freely distribute the URL identifying the publication in the public portal 


\title{
Investigation of Deep Indoor NB-IoT Propagation Attenuation
}

\author{
Krzysztof Mateusz Malarski, Jakob Thrane, Markus Greve Bech, Kamil Macheta, \\ Henrik Lehrmann Christiansen, Martin Nordal Petersen and Sarah Ruepp \\ DTU Fotonik \\ Technical University of Denmark \\ Kgs. Lyngby, 2800 DK \\ \{krmal, jathr, s144065, s150763, hlch, mnpe, srru\}@fotonik.dtu.dk
}

\begin{abstract}
Reliable connectivity over large distances is one of the main features characterising Low-Power Wide Area Network (LP-WAN) technologies, with Narrowband Internet of Things (NB-IoT) as the most promising one. The advent of such a family of communication standards has attracted attention of the industry, as excellent coverage of LP-WAN potentially enables new opportunities of automation, for instance remote metering or asset tracking. However, telecommunication standardisation bodies are still lacking accurate models of signal attenuation in deep-indoor environments. This work attempts to address this gap by presenting experimental measurements on NB-IoT deepindoor signal attenuation. The results indicate the differences in signal propagation between underground and above-ground indoor scenarios.

Index Terms-Deep indoor, NB-IoT, Path loss, Signal propagation
\end{abstract}

\section{INTRODUCTION}

The emergence of Internet Of Things (IoT) communication have paved the way for innovations that build on the automation of industrial and everyday life processes. The legacy mobile network infrastructure is not capable of handling the increased demand on the number of connected devices, however such novel solutions are expected realised within the deployment of 5G cellular systems [1], It is predicted that the amount of IoT devices deployed by year 2020 will exceed the number of personal devices by 50 times. Thanks to the advent of a new subset of IoT, LP-WAN technologies, not only massive deployments is a possibility, but also the increased link budget of LP-WAN has made it feasible to deploy applications requiring seamless connectivity under extreme radio conditions. In so called deep-indoor scenario, the base station is separated from the end-device by a multitude of solid obstacles, such as concrete walls or floors, significantly degrading the strength of the signal. Examples of deep-indoor applications can for instance be remote readings of water or gas meters, located in the basements of the building, or telemedical patient at home monitoring.

NB-IoT is a LP-WAN technology developed by 3rd Generation Partnership Project (3GPP) for IoT. The technology is aimed towards the use in existing cellular deployments using licensed spectrum for transmission, thus reducing the need for deployment of new infrastructure as is the case with similar narrowband radio technologies such as Long Range
(LoRa) and Sigfox. NB-IoT is applicable in a selection of known LTE bands ranging as low as band $28(\sim 700 \mathrm{MHz})$ to band $1(\sim 2100 \mathrm{MHz})$, thus a broad range of frequencies can be used for transmission. The range of transmission frequencies have been subject to much research through the past decades. This has resulted in accurate channel models for different transmission scenarios, which are essential for predicting coverage and capacity. Most known are singleslope path loss models such as Okumura Hata, however, a continuous effort has been made by the wireless community to improve such models for a large range of frequencies and transmission scenarios. Recently efforts have been made by International Telecommunication Union (ITU) and 3GPP to standardise and summarise the large quantity of channel models. This has more specifically resulted in such channel models as 3GPP 38.901 [2] and ITU M.2412 [3] which cover a large range of transmission scenarios in Outdoor-to-Outdoor $(\mathrm{O} 2 \mathrm{O})$ such as Dense Urban or Rural areas, but also in terms of Outdoor-to-Indoor (O2I) and Indoor-to-Indoor (I2I) scenarios for a broad range of frequencies and bandwidths.

The authors in [4] claim that excellent connectivity can be achieved in indoor environments and that IoT application requirement can be satisfied using NB-IoT. The authors in [5] show the temporal link dynamic of indoor NB-IoT devices and conclude that NB-IoT is capable of satisfying the requirements put forward for IoT applications.

The modelling of indoor coverage is difficult and remains rigid in approximations. Margins of loss, in terms of $\mathrm{dB}$, are traditionally included in link budgets to accommodate for high loss areas. This along with increased receiver sensitivity of such IoT devices allow for improved transmission ranges of many kilometres. However, margins are still included in such calculation which makes it difficult to determine and optimise for indoor deployment scenarios and especially deep indoor scenarios such as basements. Models to account for single outer wall penetration loss and other O2I losses are included in the latest 3GPP 38.901. However, the models remain too simplified for complex deep indoor situations for which additional measurements are needed. The authors in [6] explore this with the implementation of a ray-tracing model, and conclude ubiquitous coverage in deep indoor/underground areas cannot be achieved. 


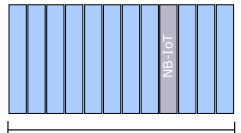

LTE carriers

In-band

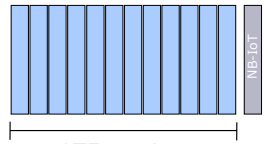

LTE carriers

Guardband

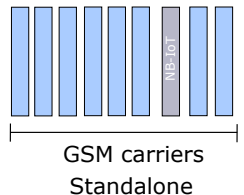

Standalone
Fig. 1. NB-IoT deployment options

It is thus of interest to further investigate such scenarios by measurements. Such studies can be additionally motivated by the fact that some of the critical IoT use cases, such as telemedicine or tracking of valuable assets, require a reliable long-range communication infrastructure to operate properly. Quality of Service guarantees and high availability are necessary also in challenging radio conditions, as the tracked objects or monitored patients may move to a hard-to-reach localisation, such as a basement. Therefore, deriving accurate channel models for deep-indoor propagation is a big step towards the realisation of critical IoT.

\section{Contributions}

In this work, we present deep indoor measurements done at 820.5 MHz (band 20 guardband) using NB-IoT. We furthermore present the following contributions

- We show how deep indoor scenarios affect received signal strength (Received Signal Strength Indicator (RSSI)).

- We analyse the effect of indoor distance to the outermost wall for different indoor scenarios.

The remainder of this paper is organised as follows. Section II introduces NB-IoT technology from the air interface perspective. The description of our field trial is included in section III, and the results are presented and discussed in section IV. Section V concludes the work.

\section{NB-IOT RADIO OVERVIEW}

In this section, we provide a brief overview of NB-IoT radio interface and its co-existence with Long-Term Evolution (LTE).

Modulation: NB-IoT uses Orthogonal Frequency-Division Multiple Access (OFDMA) for downlink and due to the same timing and bandwidth as in LTE (the bandwidth of NB-IoT is equal to $180 \mathrm{kHz}$, which corresponds to 1 LTE Physical Resource Block (PRB)) seamless co-operation is possible. In the case of uplink, NB-IoT remains compatible with LTE in single-tone mode, however, multi-tone transmission is also supported: $3.75 \mathrm{kHz}$ subcarrier spacing and $2 \mathrm{~ms}$ slot duration (LTE uses $15 \mathrm{kHz}$ spacing and $0.5 \mathrm{~ms}$ duration) [7].

Deployment options: There are three possible deployment options for NB-IoT (see Fig. 1):

1) In-band, where one of the LTE carriers is dedicated for IoT communication.

2) Guardband, where NB-IoT occupies the spectrum of a guardband of LTE.

3) Standalone, where refarming of non-LTE carriers is applied (e.g. one of the Global System for Mobile (GSM) carriers).

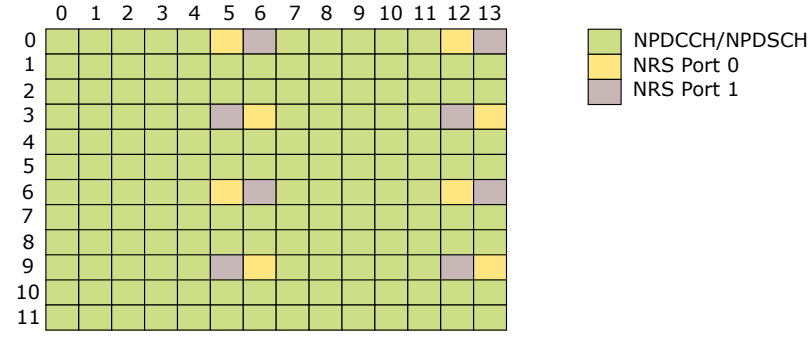

Fig. 2. NB-IoT downlink resource mapping in stand-alone and guardband modes

Consequently, NB-IoT can either be deployed separately from $4 \mathrm{G}$ network or function together with LTE as both in-band and guardband modes ensure mutual orthogonality between the technologies.

Downlink signalling and resource mapping: Downlink NBIoT transmission consists of 3 channels and 3 signals:

- Narrowband Physical Broadcast Channel (NPBCH) contains Master Information Block (MIB);

- Narrowband Physical Downlink Control Channel (NPD$\mathrm{CCH})$ carries scheduling data for both downlink and uplink;

- Narrowband Physical Downlink Shared Channel (NPDSCH) conveys the application data, paging indication and random access response;

- Narrowband Primary Synchronisation Signal (NPSS) and Narrowband Secondary Synchronisation Signal (NSSS) are used for cell discovery and synchronisation between the device and the base station;

- Narrowband Reference Signal (NRS) acts as a phase reference for downlink channels' demodulation [8].

Fig. 2 shows how NPDCCH, NPDSCH and NRS can be mapped on a PRB resource grid. Since our experiments were conducted with guardband deployment of NB-IoT, the in-band resource mapping is not presented.

\section{FIELD TRIAL}

This section explains how the experiment was prepared and conducted (setup, data collection and processing).

\section{A. Description}

The goal of the experiment was to perform NB-IoT signal strength measurements in deep-indoor locations the GPS coordinates of which were known. Then, based on the collected samples we derived a relationship between RSSI and indoor distance (i.e. the distance from the indoor reference point). The study was conducted on the campus of Technical University of Denmark in Kongens Lyngby, Denmark. We selected the rooms and the basements of 2 buildings: 343 and 340 due to their rectangular shape, available knowledge of building materials and easier access to the basements. Additionally, the measurements were taken in the campus tunnel system, which is partly at the floor level -1 , and partly at floor level -2 (see Fig. 3). Table I presents a summary of the number of acquired measurements per location. 


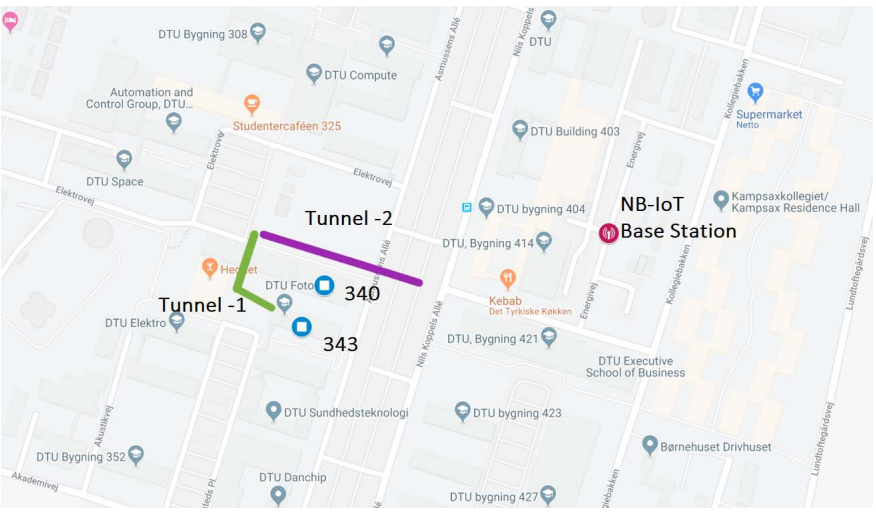

Fig. 3. Measurement locations

\begin{tabular}{|c|c|}
\hline Location & Number of samples \\
\hline Building 340 & 2172 \\
\hline Building 343 & 12256 \\
\hline Tunnel entrance & 713 \\
\hline Tunnel level -1 & 803 \\
\hline Tunnel level -2 & 6246 \\
\hline Total \# of samples & $\mathbf{2 2 1 9 0}$ \\
\hline \multicolumn{2}{|c|}{ TABLE I } \\
\hline
\end{tabular}

NUMBER OF SAMPLES PER LOCATION

\section{B. Setup}

The diagram of the experimental setup is depicted in Fig. 4. The measurements of the NB-IoT signal quality were taken as follows: the downlink signal from a neighbouring base station was captured by Rohde \& Schwartz TSMW[9] radio scanner. The setup measures RSSI and is defined according to the $3 \mathrm{GPP}$ specification. We set the device to measure at $820,5 \mathrm{MHz}$, a guardband of LTE band 20, being the frequency at which the Danish operator provides NB-IoT connectivity. TSMW was used to forward the timestamped radio information to the PC. Since it was impossible to track the position of the scanner by means of GPS (lack of deep-indoor coverage, insufficient accuracy), a system of 2 LIDAR-LITE V3HP [10] lasers and 1 Arduino Mega 2560[11] board was applied for positioning. The lasers, pointing at the walls of the room, were measuring the distances to the walls, and the Arduino board was collecting the distances and translating them into XY coordinates, which were sent to the PC with timestamps. All the devices were placed on a trolley, so that the data from different spots in the room could be taken. Fig. 5 shows the experimental setup in one of our measurement locations.

The distance from the NB-IoT transmitter to the experimental setup is $\sim 450 \mathrm{~m}$ as the crow flies, with the propagation area covered by vegetation and densely spaced brick-buildings with an average height of 2 floors.

\section{Data processing}

Raw measurements were post-processed using MATLAB. By means of timestamps, the RSSI samples from TSMW and $\mathrm{XY}$ co-ordinates from the laser setup were concatenated into one synchronised dataset consisting of esimated GPS positions

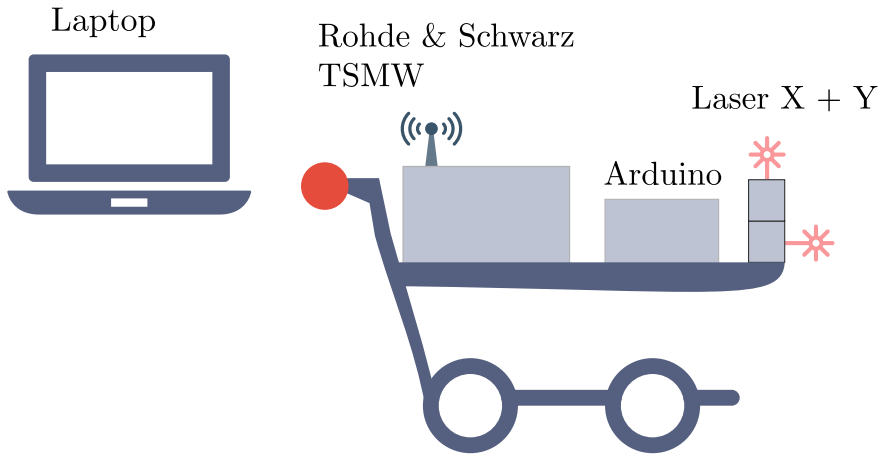

Fig. 4. Experimental setup

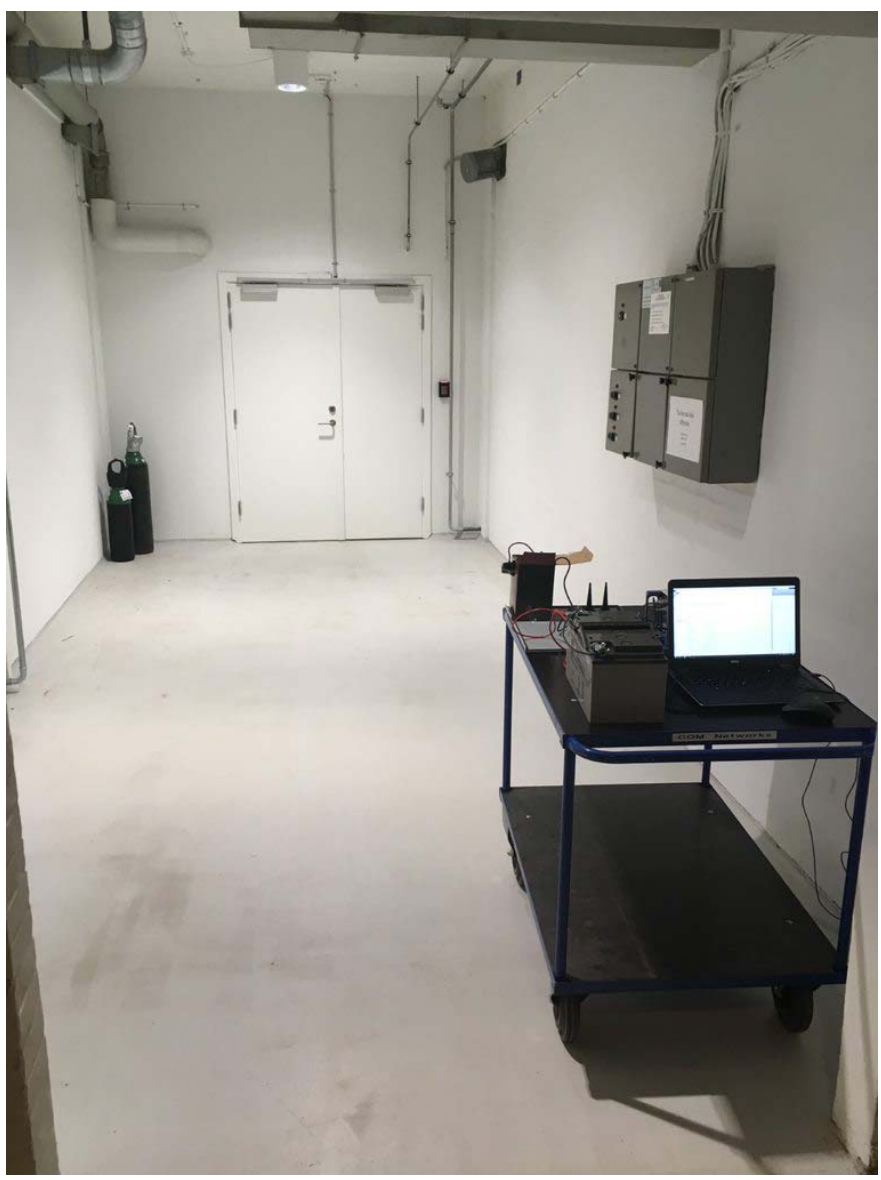

Fig. 5. Measurements in the basement of building 340

and RSSI measurements. From each GPS position an indoor distance metric is derived. This is achieved using the position of the base station and a reference point. Thus, we define the indoor distance as the distance between the measurement and the outer most wall that is closest to the transmitter location.

\section{Indoor path loss model}

Principles for path loss modelling for O2I are available in [2]. The overall path loss can be modelled by the following:

$$
P L=P L_{b}+P L_{t w}+P L_{i n}+N\left(0, \sigma_{p}^{2}\right)
$$




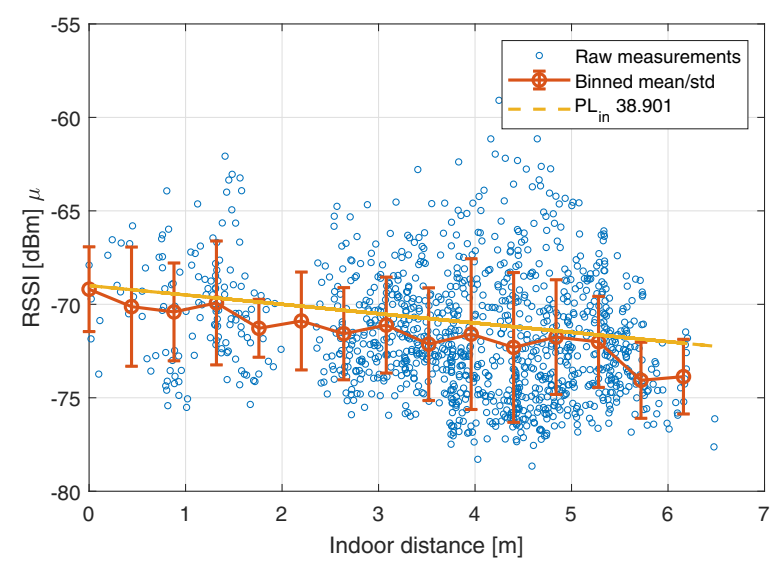

Fig. 6. RSSI (in $\mathrm{dBm}$ ) with respect to indoor distance (in metres), ground floor staircase. Indoor estimated path loss shown for comparison.

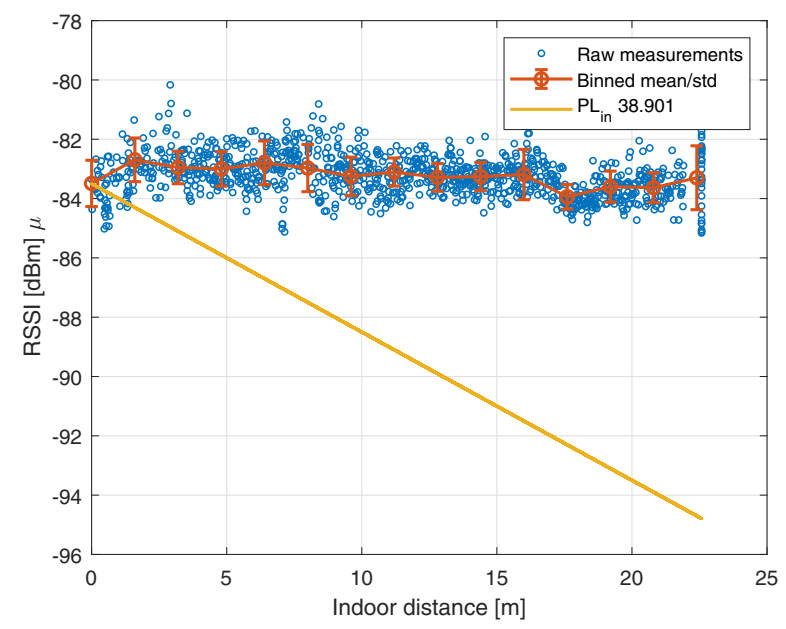

Fig. 7. RSSI (in $\mathrm{dBm}$ ) with respect to indoor distance (in metres), tunnel level -2. Indoor estimated path loss shown for comparison.

Where $P L_{b}$ is the basic outdoor path loss, $P L_{t w}$ is the building penetration loss through the external wall. $P L_{i n}$ is the loss that is dependent on the depth into the building. $\sigma_{p}^{2}$, is the standard deviation for the penetration loss.

In this work the focus is on $P L_{i n}$ and losses hereof. The proposed model in [2] is very simple and as follows:

$$
P L_{i n}=0.5 d_{2 D-i n}
$$

Where $d_{2 D-i n}$ is the indoor distance in $2 \mathrm{D}$, to the outer wall.

\section{RESUlts}

Figures 6 and 7 illustrate the relationship between the RSSI and the indoor distance for the data collected in the corridor on the ground floor of the building 343 (Fig. 6) and in the campus tunnel on the level -2 (Fig. 7). Interestingly, one can identify several differences between the plots. First of all, the strength of the signal in the underground location is generally

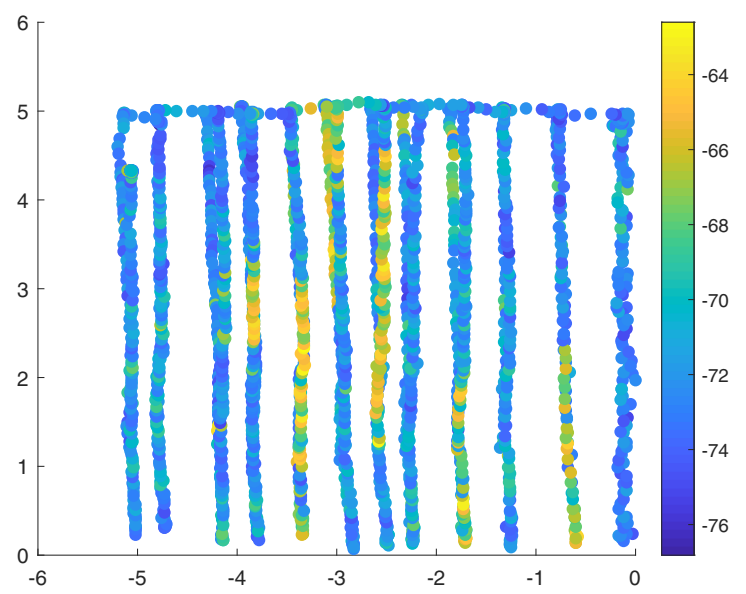

Fig. 8. Power heatmap (RSSI vs. XY position in metres), ground floor staircase

more than $13 \mathrm{dBm}$ weaker than in the building. However, while the RSSI degrades with increasing indoor distance in the ground level case, in the tunnel environment it only slightly fluctuates around the same value. Such a discrepancy can be a consequence of the fact that in the case of Fig. 6 the reference point is the end of the staircase, and with only one wall separating the place from the open-air; in the case of Fig. 7 the starting spot is the entrance to the tunnel, already separated from the outside world by multiple walls. Thus, in the -2 level the radio conditions do not worsen significantly, as in the ground floor scenario.

Fig. 8 shows how strong was the captured NB-IoT downlink signal in various spots of a laboratory in building 343. The shape of the scatter plot corresponds to the movements of the trolley with the measurement setup. It has to be mentioned that point $(0,0)$ was the corner of the room and thus the outer stripes in the figure signify the contours of the area. It can be seen that the RSSI values were generally lower in the locations adjacent to the walls, while the middle of the laboratory was characterised by the strongest signal. A different pattern can be seen in Fig. 9, for which the measurements were taken in the narrow tunnel. The strength of the signal for all samples is lower than in Fig. 8, however, the values obtained in the tunnel are very homogeneous.

It is observed in Fig. 6 that the path loss model provided by [2] for indoor-based distance loss fits the obtained data quite satisfactory. It is thus observed to be a good approximation of attenuation given indoor distance at the ground floor for NB-IoT. However, this is not the case in Fig. 7, where the measured RSSI values do not follow a linear trend of the theoretical path loss model.

\section{CONCLUSION}

In this work, we presented our experimental results of NBIoT deep-indoor signal propagation study. We prepared a sim- 


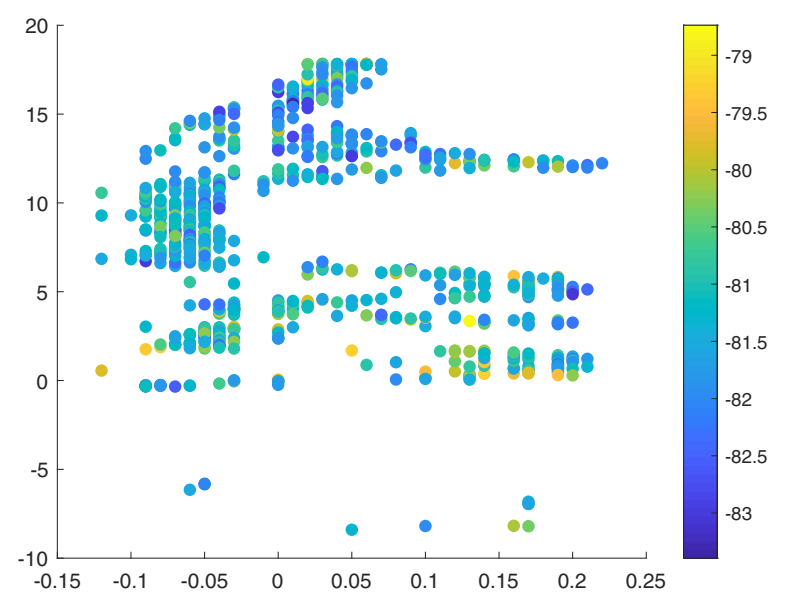

Fig. 9. Power heatmap (RSSI vs. XY position in metres), tunnel level -1

ple movable setup and perform a series of RSSI measurements in several locations on the DTU campus.

The results revealed that the indoor path loss model derived in 3GPP TR 38.901 standard fitted fairly well to our aboveground indoor scenario, however, it did not fit to the measurements done in the underground tunnel. In our case this could imply that the theoretical models are not suited for all spectrum of indoor scenarios, as the complete link budget is too complex to be accurately represented by a linear dependence. In order to verify such a hypothesis more experiments in the deepindoor environment must be performed, yet we believe that the outcome of this work can be used as input to the validation of indoor path loss models for NB-IoT use cases.

\section{ACKNOWLEDGMENTS}

This work was partially supported by Innovation Fund Denmark through the Eureka Turbo project IoT Watch4Life.

\section{REFERENCES}

[1] M. R. Palattella, M. Dohler, A. Grieco, G. Rizzo, J. Torsner, T. Engel, and L. Ladid, "Internet of Things in the 5G Era: Enablers, Architecture, and Business Models," IEEE Journal on Selected Areas in Communications, vol. 34, no. 3, pp. 510-527, mar 2016. [Online]. Available: http://ieeexplore.iee.org/document/7397856/

[2] TSGR, "TR 138901 - V14.3.0 - 5G; Study on channel model for frequencies from 0.5 to $100 \mathrm{GHz}$ (3GPP TR 38.901 version 14.3.0 Release 14)." [Online]. Available: http://www.etsi.org/standards-search

[3] Itu-r, "Guidelines for evaluation of radio interface technologies for IMT2020 M Series Mobile, radiodetermination, amateur and related satellite services." [Online]. Available: http://www.itu.int/ITU-R/go/patents/en

[4] S. Z. Khan, H. Malik, J. L. Redondo Sarmiento, M. M. Alam, and Y. L. Moullec, "DORM: Narrowband IoT Development Platform and Indoor Deployment Coverage Analysis," Procedia Computer Science, vol. 151, pp. 1084-1091, jan 2019. [Online]. Available: https://www.sciencedirect.com/science/article/pii/S1877050919306210

[5] S. P. Hassan Malik, Muhammad Sikandar Zulqarnain Khan, Jeffrey Leonel Redondo Sarmiento, Muhammad Mahtab Alam, Yannick Le Moullec and Arand, "NB-IoT Network Trial : Indoor, Outdoor and Underground Coverage Campaign," no. April, 2019.

[6] S. Gangakhedkar, Ö. Bulakci, and J. Eichinger, "Addressing deep indoor coverage in narrowband-5G," IEEE Vehicular Technology Conference, vol. 2017-Septe, no. Mcl, pp. 1-5, 2018.
[7] N. Mangalvedhe, R. Ratasuk, and A. Ghosh, "NB-IoT deployment study for low power wide area cellular IoT," in 2016 IEEE 27th Annual International Symposium on Personal, Indoor, and Mobile Radio Communications (PIMRC). IEEE, sep 2016, pp. 1-6. [Online]. Available: http://ieeexplore.iee.org/document/7794567/

[8] J. Schlienz and D. Raddino, "Narrowband Internet of Things Whitepaper NarrowBand_IoT 1MA266_0e," Tech. Rep. [Online]. Available: www.rohde-schwarz.com/appnote/

[9] U. Manual, "R\&S TSMW Universal Radio Network Analyzers User Manual," Tech. Rep., 2017.

[10] "Garmin LIDAR-Lite v3HP — Distant Measurement Sensor."

[11] "Arduino Mega 2560 Rev3." 